\title{
Chiral Symmetry Breaking in Micro-Ring Optical Cavity By Engineered Dissipation
}

\author{
Fang-Jie Shu, ${ }^{1,2,3}$ Chang-Ling Zou, ${ }^{2,4,5}, *$ Xu-bo Zou, ${ }^{2,4}$ and Lan Yang ${ }^{3}$ \\ ${ }^{1}$ School of Physics and Electrical Information, Shangqiu Normal University, Shangqiu, Henan 476000, China \\ ${ }^{2}$ Key Laboratory of Quantum Information, University of Science and Technology of China, CAS, Hefei, Anhui 230026, China \\ ${ }^{3}$ Electrical and Systems Engineering Department, \\ Washington University, St. Louis. Missouri 63130, USA \\ ${ }^{4}$ Synergetic Innovation Center of Quantum Information $\&$ Quantum Physics, \\ University of Science and Technology of China, Hefei, Anhui 230026, China \\ ${ }^{5}$ Department of Applied Physics, Yale University, New Haven, Connecticut 06511, USA
}

\begin{abstract}
We propose a method to break the chiral symmetry of light in traveling wave resonators by coupling the optical modes to a lossy channel. Through the engineered dissipation, an indirect dissipative coupling between two oppositely propagating modes can be realized. Combining with reactive coupling, it can break the chiral symmetry of the resonator, allowing light propagating only in one direction. The chiral symmetry breaking is numerically verified by the simulation of an electromagnetic field in a micro-ring cavity, with proper refractive index distributions. This work provokes us to emphasize the dissipation engineering in photonics, and the generalized idea can also be applied to other systems.
\end{abstract}

\section{INTRODUCTION}

Usually, traveling wave resonators, such as whisperinggallery (WG) microcavities [1-3], hold the mirror reflection geometry symmetry and support clockwise (CW) and counter clockwise (CCW) traveling wave modes simultaneously. Because of time-reverse symmetry of light, $\mathrm{CW}$ and CCW modes degenerate in non-gyromagnetic materials. When there is external perturbation to the cavity boundary, the $\mathrm{CW}$ and CCW modes will couple to each other and form a pair of standing wave modes, which are odd and even symmetric superpositions of CW and CCW waves with equal weights $[4,5]$. In consequence, transmission of a passive cavity or the emission of an active cavity are symmetric in both directions. Although this feature has no harm for most applications like narrow-band filters, optical resonators with chiral symmetry breaking are of great interest in both physics and many applications including unidirectional lasers [6].

Several approaches have been proposed to break the chiral symmetry of a microcavity system. One category of approaches addresses the issue by deforming a microcavity to break the chiral symmetry in terms of geometry. People can create deformed microcavities with a chiral boundary shape, such as a spiral micro-cavity $[7,8]$ and an asymmetric limacon micro-cavity [9], to realize unidirectional emission of light. A circular cavity can also be tuned to a chiral one with multiple scatterers on the rim [10-14], which will benefit microcavity-based nanoparticle sensing. In another category, a rotation $[15,16]$ or a time dependent modulation [17-19] along angular direction of a microcavity introduces an effective angular momentum, breaking the time reversal symmetry of light in the WG microcavity. Furthermore, both the geometry and time symmetry are broken by rotating a limacon

\footnotetext{
* Corresponding author: clzou321@ustc.edu.cn
}

cavity [20], which provides a sensitive on-chip rotation detector by monitoring far-field emission patterns.

In this paper, we propose an alternative approach to break the chiral symmetry in a micro-ring cavity, based on the engineered dissipation [21-24] of optical modes. By balancing the direct reactive coupling and indirect dissipative coupling with proper phase difference, the cavity eigenmodes become pure $\mathrm{CW}$ or $\mathrm{CCW}$, which are the chiral modes in a micro-ring. To verify the theory, we introduce dielectric perturbations to a normal micro-ring, that is, adding pure real or imaginary dielectric constant onto original one.

The numerical simulation based on the Maxwell equations confirms the prediction of our analytical model. The chiral eigenmodes and an asymmetric transmission spectra in forward and backward directions are shown in the micro-ring system. We believe this simple and dissipative-related asymmetry micro-ring can be used as a laser resonator with unidirectional emission and as a key element of a highly sensitive sensor [25, 26].

\section{PRINCIPLE}

Before a detailed study on the WG modes, we briefly discuss a general model of reactive and dissipative interactions between bosonic modes. Suppose there are two ordinary mutual coupled modes $a$ and $b$ coupling with a lossy mode $c$, then the coupled mode equations described the three mode system are (see Fig. 1(a) left penal)

$$
\frac{d}{d \zeta}\left(\begin{array}{c}
a \\
b \\
c
\end{array}\right)=\left(\begin{array}{ccc}
\chi_{a} & i g_{a b} & i g_{a c} \\
i g_{a b} & \chi_{b} & i g_{b c} \\
i g_{a c} & i g_{b c} & \chi_{c}
\end{array}\right)\left(\begin{array}{l}
a \\
b \\
c
\end{array}\right) .
$$

Here, the system evolves along the general axis $\zeta$, which can be time, space or other generalized coordinates. In the parameter $\chi_{x}=-i \omega_{x}-\kappa_{x}, \omega_{x}$ and $\kappa_{x}$ are intrinsic oscillation frequencies and rates of dissipation to independent channels, respectively. They vary with coordinate $\zeta$ 


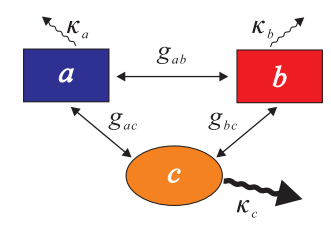

(a)
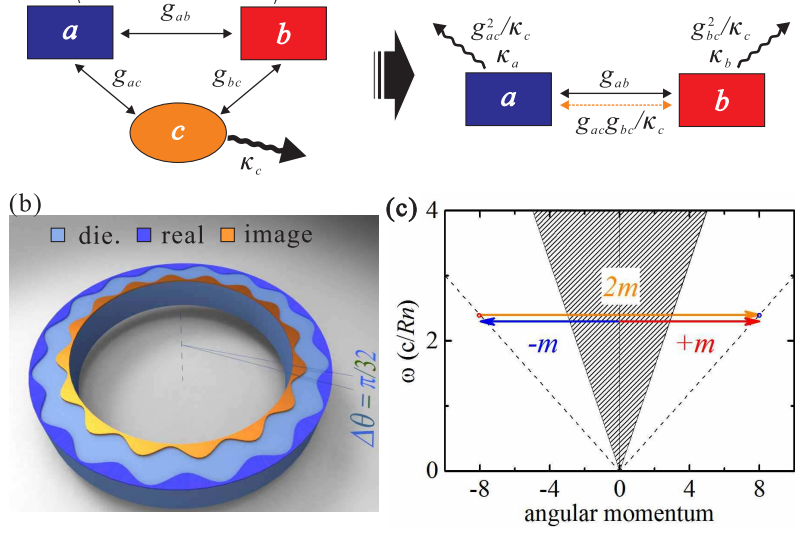

Figure 1. (Color online) (a) A schematic illustration of a dissipative coupling by an engineered dissipation. The modes $a$ and $b$ couple to a common lossy channel $c$ (left penal). It can be reduced to a effective model in which $a$ and $b$ dissipatively coupled with each other (right penal). (b) A micro-ring with dielectric and absorptive materials depositing or doping on each rims works as a pure chiral resonator. (c) A phase matching diagram for modes with mode index $m= \pm 8$.

for mode $x \in\{a, b, c\}$. The $g_{a b}, g_{a c}$ and $g_{b c}$ are the common reactive couplings between modes $\mathrm{a}, \mathrm{b}$, and $\mathrm{c}$, which implies the coupling inducing a coherent energy transfer between the modes. If $\kappa_{c} \gg \max \left(\kappa_{a}, \kappa_{b}, g_{a b}, g_{a c}, g_{b c}\right)$ and $\kappa_{c} \gg \max \left(\omega_{c},\left|\omega_{c}-\omega_{a}\right|,\left|\omega_{c}-\omega_{b}\right|\right)$ are satisfied, i.e. relaxation rate of the mode $c$ is ultra-fast and the mode $c$ is approximately at the static state $\frac{d}{d \zeta} c=0$. Therefore, $c \approx \frac{i g_{c a}}{\kappa_{c}} a+\frac{i g_{c b}}{\kappa_{c}} b$ and we arrive at the effective coupling modes equations (Fig. 1(a) right penal)

$$
\frac{d}{d \zeta}\left(\begin{array}{l}
a \\
b
\end{array}\right)=\left(\begin{array}{cc}
\chi_{a}-\frac{g_{a c}^{2}}{\kappa_{c}} & i g_{a b}-\frac{g_{a c} g_{b c}}{\kappa_{c}} \\
i g_{a b}-\frac{g_{a c} g_{b c}}{\kappa_{c}} & \chi_{b}-\frac{g_{b c}^{2}}{\kappa_{c}}
\end{array}\right)\left(\begin{array}{l}
a \\
b
\end{array}\right) .
$$

As expected, the introduced lossy channel to modes $a$ and $b$ induces additional dissipations $\frac{g_{a c}^{2}}{\kappa_{c}}$ and $\frac{g_{b c}^{2}}{\kappa_{c}}$, respectively. However, it is not intuitive that there is an additional term $\frac{g_{a c} g_{c b}}{\kappa_{c}}$ for the coupling between the two modes, due to the coherent interference in the shared lossy channel. The additional coupling term is a real number, which indicates that it is a dissipative coupling instead of a reactive coupling.

If the the coupling to bath $\left(g_{a c, b c}\right)$ and the internal coupling $\left(g_{a b}\right)$ are modulated along $\zeta$, the effective coupling between $a$ and $b$ can be rewrote as complex function of $\zeta$

$$
g_{\text {eff }}(\zeta)=\left|g_{\text {eff }}(\zeta)\right| e^{i \varphi(\zeta)}
$$

The nontrivial phase accumulation $\varphi(\zeta)$ will lead to interesting effects, which are absent in a system of pure reactive or dissipative coupling. In this paper, we focus on a specially engineered dissipation in which $\varphi(\zeta)=\Omega \zeta$ is a linear equation of $\zeta$. As a consequence, the effective coupling $\left|g_{\text {eff }}(\zeta)\right| e^{i \Omega \zeta}$ includes a generalized momentum $\Omega$, which breaks $\zeta$-symmetry of a system.

\section{CHIRAL SYMMETRY BREAKING}

Now, we apply the principle to the WG micro-ring as depicted in Fig. 1(b), where $\zeta$ denotes an angle $\theta$. Mode amplitudes in WG micro-ring evolve as

$$
\frac{d}{d \theta} a_{m}=\left(-i m-\frac{m}{2 Q}\right) a_{m}=\chi_{m}^{(\theta)} a_{m}
$$

where $m$ is an integer corresponding to the angular momentum (mode index) of the mode, and $Q$ is the quality factor of the mode. The modes with positive and negative $m$ have a degenerate mode frequency but opposite propagation directions, which are called clockwise (CW) and counter-clockwise (CCW) modes. To obtain an effective coupling between $\mathrm{CW}$ and CCW modes with the non-zero momentum $\Omega$, extra materials distributed sinusoidally on the inner and the outer rims are constructed, noting that there is a shifted angle $\Delta \theta$ between these two perturbations (Fig. 1(b)). The reactive coupling between traveling wave modes is controlled by external refractive index perturbation (dark blue part on the micro-ring in Fig. 1(b)), such as a dielectric pattern on the surface of the micro-ring. The dissipative coupling can be introduced by putting lossy nanoparticle or doping absorptive atom on the cavity (orange part on the micro-ring in Fig. 1(b)), which induces losses to CW and CCW modes simultaneously.

We can formalize the effective dielectric perturbation as

$$
\begin{aligned}
\delta \varepsilon(\theta) & =\delta \varepsilon_{r}[\cos \Omega(\theta+\Delta \theta)+1]+i \delta \varepsilon_{i}(\cos \Omega \theta+1) \\
& =\delta \varepsilon_{+} e^{i \Omega \theta}+\delta \varepsilon_{-} e^{-i \Omega \theta}+\delta \varepsilon_{0},
\end{aligned}
$$

where $\delta \varepsilon_{+}=\frac{\delta \epsilon_{r} e^{i \phi}+i \delta \epsilon_{i}}{2}, \delta \varepsilon_{-}=\frac{\delta \varepsilon_{r} e^{-i \phi}+i \delta \varepsilon_{i}}{2}, \delta \varepsilon_{0}=$ $\delta \varepsilon_{r}+i \delta \varepsilon_{i}$ and $\phi=\Omega \Delta \theta$. Due to the phase matching condition, the term $e^{i \Omega \theta}$ scatters the light in the mode $m$ to the mode $m+\Omega$. For example, in Fig. 1 (b) we have a created momentum $\Omega=16$ by engineering the dissipative coupling. This coupling couples $m=-8$ mode to $m=8$ mode ((Fig. 1(c))). It is worth noting that besides the phase matching condition, energy conservation is another criterion for effective coupling. As a result, mode coupling occurs between degeneracy modes with a same $|m|$. For example, the phase matching condition between $m=-7$ and $m=9$ is satisfied, but their mode frequencies are different by two free spectra ranges (FSR), which is many orders of magnitude larger than the intrinsic linewidth of the high- $Q$ WG modes. So they cannot couple with each other in this way.

In the following, we take the $m= \pm 8$ modes in the micro-ring (Fig. 1(b)) as an example, and denote the CW and CCW modes with $a_{+}$and $a_{-}$for simplicity. The evolution of two modes, a specific form of the Eq. (2), can be written as

$$
\frac{d}{d \theta}\left(\begin{array}{c}
a_{+} \\
a_{-}
\end{array}\right)=\left(\begin{array}{cc}
\chi_{m}^{(\theta)}-\varrho \delta \varepsilon_{0} & \left.\varrho \delta \varepsilon_{+}\right) \\
\varrho \delta \varepsilon_{-} & \chi_{-m}^{(\theta)}-\varrho \delta \varepsilon_{0}
\end{array}\right)\left(\begin{array}{c}
a_{+} \\
a_{-}
\end{array}\right) .
$$


(a)

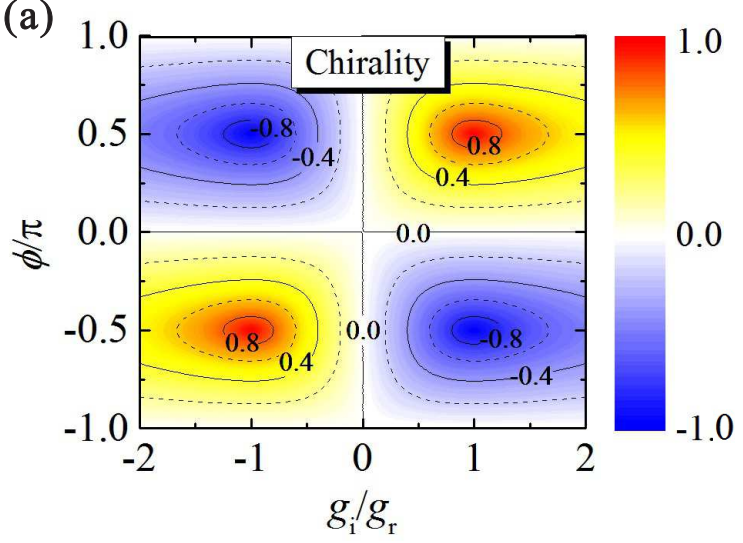

(c)

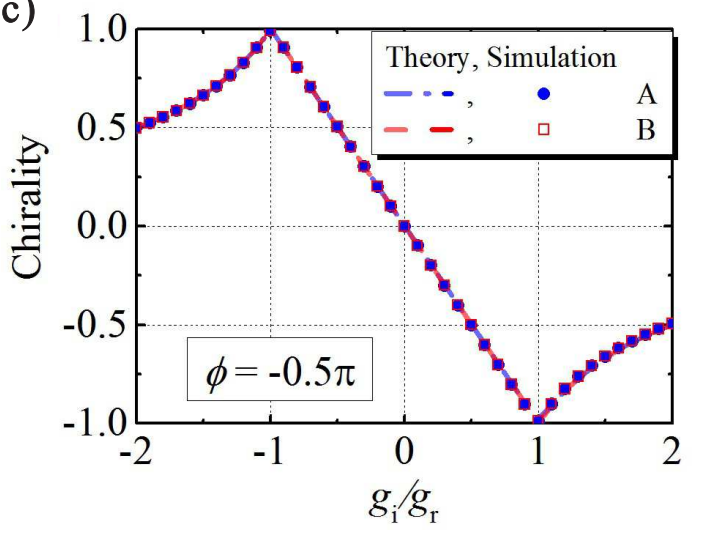

(b)

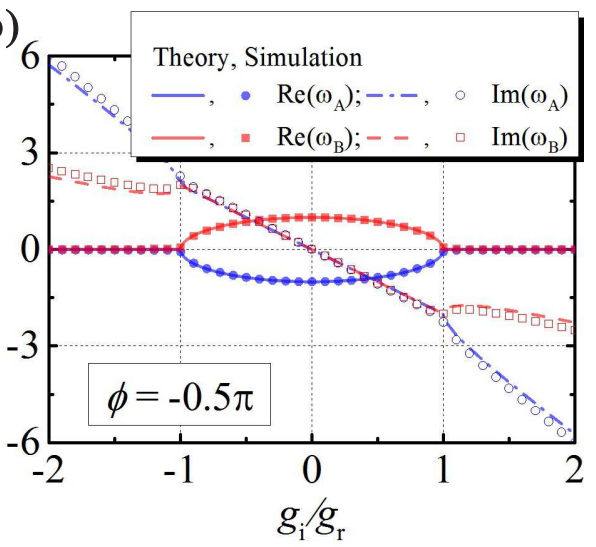

(d)

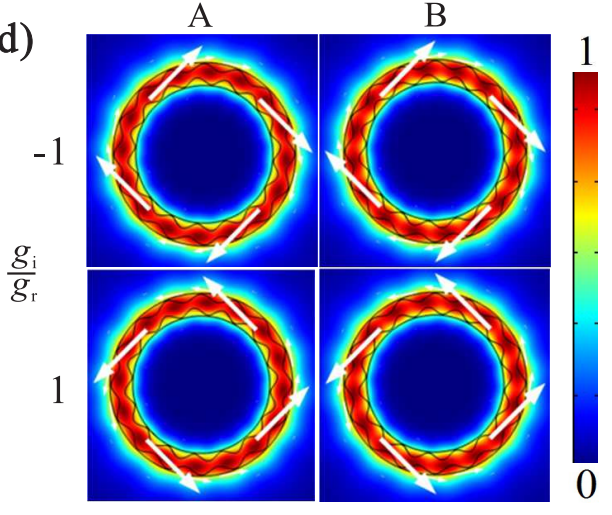

Figure 2. (Color online) (a) A chirality $K$ as a function of $g_{i} / g_{r}$ and $\phi$. The frequencies (b) and chirality (c) of eigenmodes in the chiral microresonator with $\phi=-\frac{\pi}{2}$. The lines are analytical results, and dots are from numerical simulations. (d) The mode field presents with modulus of electric field intensity distributions of $K=+1$ (first row) and -1 (last row), where white arrows indicate the Poynting vectors.

Here, perturbation effect is proportional to the $\delta \varepsilon(\theta)$ with a coefficient $\varrho$. Since the light traveling in the microring is with periodic boundary condition, we translate the coordinate $\theta$ to $t=\tau_{r} \theta / 2 \pi$ for convenience, where the $\tau_{r}$ is the round-trip time of the cavity. Then, in a rotating frame of cavity frequency, the temporal evolution becomes

$$
\frac{d}{d t}\left(\begin{array}{c}
a_{+} \\
a_{-}
\end{array}\right)=\left(\begin{array}{cc}
-\kappa-2 g_{i} & i g_{r} e^{i \phi}-g_{i} \\
i g_{r} e^{-i \phi}-g_{i} & -\kappa-2 g_{i}
\end{array}\right)\left(\begin{array}{c}
a_{+} \\
a_{-}
\end{array}\right),
$$

where $g_{r}$ and $g_{i}$ are the reactive and dissipative coupling strength, respectively.

The normal modes of the coupling system can be solved as

$$
A, B=\frac{ \pm p a_{+}+a_{-}}{\sqrt{1+|p|^{2}}},
$$

where

$$
p=\frac{i e^{i \frac{\phi}{2}} \sqrt{\left(i g_{r}-g_{i} e^{i \phi}\right)\left(g_{i}-i g_{r} e^{i \phi}\right)}}{i g_{r}-g_{i} e^{i \phi}} .
$$

The eigenfrequencies are

$$
\omega_{A, B}=-i 2 g_{i} \mp e^{-i \frac{\phi}{2}} \sqrt{\left(i g_{r}-g_{i} e^{i \phi}\right)\left(g_{i}-i g_{r} e^{i \phi}\right)} \cdot(10)
$$

From the Eq. (8), the ingredients of $\mathrm{CW}$ and $\mathrm{CCW}$ circulating light in the new normal modes are generally not balanced. For both normal modes, the portion of CCW light is $\frac{1}{1+|p|^{2}}$. Here, we define the chirality of the system as

$$
K=\frac{I_{C W}-I_{C C W}}{I_{C W}+I_{C C W}}=\frac{|p|^{2}-1}{|p|^{2}+1},
$$

where $I_{C W}\left(I_{C C W}\right)$ is energy of $\mathrm{CW}(\mathrm{CCW})$ ingredient.

In Fig. 2(a), the chirality $K$ of the modes are theoretically calculated with respect to relative coupling strengths $\frac{g_{i}}{g_{r}}$ and phase differences $\phi$. It is clearly shown that $K=0$ if $g_{i}$ or $\phi$ is zero, and high chirality is obtained near the points $( \pm 1, \pm \pi / 2)$. Specially, if we choose $\phi=-\frac{\pi}{2}$, analytical results will be simplified as $p=i \sqrt{\frac{g_{i}}{g_{r}}-1} / \sqrt{\frac{g_{i}}{g_{r}}+1}$. As shown in Fig. 2(b), the system behaviors have two regions separated by points $\left|\frac{g_{i}}{g_{r}}\right|=1$. In region $\frac{g_{i}}{g_{r}} \in[-1,1]$, the frequency splits, i.e. $\operatorname{Re}\left(\omega_{A}-\omega_{B}\right) \neq 0$, while the imaginary part are the same $-i 2 g_{i}$, thus the couplings only change the frequencies of the uncoupled degenerated $\mathrm{CW}$ and $\mathrm{CCW}$ modes. For parameter $\frac{g_{i}}{g_{r}}$ outside the interval, $\omega_{A, B}$ are pure imaginary numbers, which removes the degeneracy by adding 
different losses. Remarkably, for $\frac{g_{i}}{g_{r}}=1\left(\frac{g_{i}}{g_{r}}=-1\right)$, the system only supports pure CCW (CW) mode as $K=-1$ $(+1)$ (Fig. 2(c)).

\section{NUMERICAL RESULTS}

To check the validity of the idea about breaking chiral symmetry in traveling wave optical resonators by engineering the dissipation, we solve out eigenmodes and reflection spectra in those cavities numerically. Such numerical verification is necessary, because the principle and model in previous section are idealized. In realistic systems, there should be multiple modes, and the coupled mode equations are just results of perturbation theory about the system, these approximations may lead to unpredicted effect to the chiral symmetry breaking.

In the following, the model proposed in Fig. 1(b) is simulated by finite element method (COMSOL multiphysics). In the simulation, only the geometry and the dielectric constant of the ring distribution are set in the software. The eigenmodes and propagations of light are obtained by using the numerical method to solve the Maxwell equations, without any assumptions. Therefore, the numerical results in the following part will provide an independent and experiment-feasible verification to our theory.

\section{A. Eigenfrequencies and Chirality}

The geometry of model in COMSOL is the same as that in Fig. 1(b), but simplified in two-dimensional model. In this model, the radii of inner and outer circle are $0.6 \mu \mathrm{m}$ and $0.8 \mu \mathrm{m}$, respectively, so the width of the ring is $0.2 \mu \mathrm{m}$. In addition, amplitudes of both sinusoidal curves are $0.03 \mu \mathrm{m}$, and shift $0.01 \mu \mathrm{m}$ away from rims to avoid sharp structure which arises singularity in the simulation. In the material side, the dielectric constant of the main body of the ring is $\epsilon=10$. The inner and outer parts sinusoidal curves inclosed dielectric perturbation $(\delta \epsilon)$ of imaginary $\left(\delta \epsilon_{i}\right)$ and real $\left(\delta \epsilon_{r}\right)$ part, respectively. The $\delta \epsilon_{i}$ and the $\delta \epsilon_{r}$ are three orders of magnitude less than $\epsilon$ to insure the validation of the perturbation approximation. As mentioned in the above analysis, the coupling strength $g_{i}\left(g_{r}\right)$ should be proportional to the perturbation $\delta \epsilon_{i}$ $\left(\delta \epsilon_{r}\right)$ [27]. Due to the asymmetric field distribution in the inner and outer rim of the micro-ring resonator, the balanced reactive and dissipative couplings are achieved with $\delta \epsilon_{i}=0.01$ and $\delta \epsilon_{r}=0.005175$. It should be noticed that the sign of $\delta \epsilon_{i}$ determines whether the materials loss $(+)$ or gain $(-)$. The sign of $\delta \epsilon_{i}$ changes easily either in theory or in simulation, but in practical experiments loss is preferred for convenience.

We performed the simulation with fixed $\phi=-\frac{\pi}{2}$ and $\delta \epsilon_{r}=0.005175$ but varies $\delta \epsilon_{i}$ from 0.02 to -0.02 corresponding to that $\frac{g_{i}}{g_{r}}$ varies from -2 to 2 . To compare with the theory, the numerical results of $\omega$ are drawn in
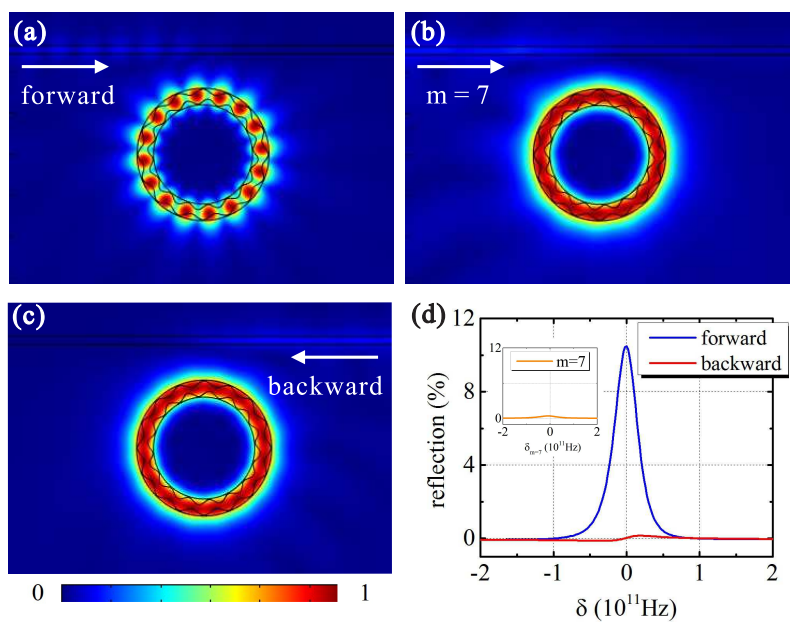

Figure 3. (Color online) (a) Forward coupling in critical coupling region with $\delta=0$. A back scattered wave forms a standing wave in the cavity. (b) Forward coupling of $m=7$ mode. (c) Backward coupling. The false color represents modulus of electric field intensity. (d) Spectrum of reflections $R$ in aforementioned situations.

coordinate system with original point $\left(\omega_{A}-\omega_{B}\right) / 2$ and frequency unit $\left|\operatorname{real}\left(\omega_{A}-\omega_{B}\right)\right| / 2$, where $\omega_{A}$ and $\omega_{B}$ are evaluated at $g_{i}=0$. It is shown in Fig. 2(b) that numerical results (symbols) are well consistent with analytic one (line). In addition, the chirality can calculated numerically by

$$
K= \pm 2 \frac{E_{\max } E_{\min }}{E_{\max }^{2}+E_{\min }^{2}}
$$

where $E_{\min }$ and $E_{\max }$ are minimum and maximum of electric field modules along the path $r=0.72 \mu \mathrm{m}$, (Fig. $2(\mathrm{c}))$. The prior circulate direction or sign of chirality is known from Poynting vectors (white arrows in Fig. $2(\mathrm{~d})$ ). The mode distributions at point $\frac{g_{i}}{g_{r}}=1$ (Fig. 2(d) lower panel) show remarkable $\mathrm{CCW}$ traveling mode with $K \approx-1$. The reverse $\mathrm{CW}$ mode can also be achieved at $\frac{g_{i}}{g_{r}}=-1$ (Fig. 2(d) upper panel) or at $\phi=\frac{\pi}{2}$ (Fig. 2(a)).

\section{B. Reflection and Transmission Spectrum}

The chirality can also be checked by sending probe laser to the cavity and measuring the reflectivity and transitivity of the cavity. As shown in Fig. 3, by putting a straight dielectric waveguide in the vicinity of the microring, light from either left or right port in waveguide can couple with the modes in micro-ring. Suppose that there are input signals $E_{i n}^{+}=a_{i n}^{+} e^{-i \omega_{i n} t}$ and $E_{i n}^{-}=a_{i n}^{-} e^{-i \omega_{i n} t}$ couple to $\mathrm{CW}$ and $\mathrm{CCW}$ modes in the cavity, respec- 
tively. The coupling mode equations become

$$
\begin{aligned}
\frac{d a_{+}}{d t}= & \left(-i \omega_{0}-\kappa-2 g_{i}-\kappa_{i n}\right) a_{+}+\left(i g_{r} e^{i \phi}-g_{i}\right) a_{-} \\
& +\sqrt{2 \kappa_{i n}} a_{i n}^{+} e^{-i \omega_{i n} t}, \\
\frac{d a_{-}}{d t}= & \left(-i \omega_{0}-\kappa-2 g_{i}-\kappa_{i n}\right) a_{-}+\left(i g_{r} e^{-i \phi}-g_{i}\right) a_{+} \\
& +\sqrt{2 \kappa_{i n}} a_{i n}^{-} e^{-i \omega_{i n} t} .
\end{aligned}
$$

We focus on the pure CCW microcavity with $\frac{g_{i}}{g_{r}}=1$ and $\phi=-\frac{\pi}{2}$. With the input-output relations $a_{\text {out }}^{+}=$ $a_{\text {in }}^{+}-\sqrt{2 \kappa_{\text {in }}} a_{+}, a_{\text {out }}^{-}=a_{\text {in }}^{-}-\sqrt{2 \kappa_{\text {in }}} a_{-}$, the forward steadystate transmissions (coincident with $\mathrm{CW}, a_{i n}^{+} \neq 0$ ) and the backwards ones at critical coupling $\kappa_{i n}=\kappa+2 g_{i}=\gamma$ and detuning $\delta=\omega_{i n}-\omega_{0}$ are

$$
\begin{aligned}
& T_{+}=\left|\frac{a_{\text {out }}^{+}}{a_{\text {in }}^{+}}\right|^{2}=\frac{\delta^{2}}{\delta^{2}+4 \gamma^{2}}, \\
& T_{-}=\left|\frac{a_{\text {out }}^{-}}{a_{\text {in }}^{-}}\right|^{2}=\left|\frac{i \delta}{i \delta-2 \gamma}+\frac{a_{\text {in }}^{+}}{a_{\text {in }}^{-}} \frac{2 g_{r} 2 \gamma}{(i \delta-2 \gamma)^{2}}\right|^{2} .
\end{aligned}
$$

They are typical Lorentz line shapes as same as those in a symmetric microcavity when only one input port has an input signal. The reflections are

$$
\begin{aligned}
& R_{+}=\left|\frac{a_{\text {out }}^{-}}{a_{\text {in }}^{+}}\right|^{2}=\left|\frac{a_{i n}^{-}}{a_{i n}^{+}} \frac{i \delta}{i \delta-2 \gamma}+\frac{2 g_{r} 2 \gamma}{(i \delta-2 \gamma)^{2}}\right|^{2} \\
& R_{-}=\left|\frac{a_{\text {out }}^{+}}{a_{-i n}}\right|^{2}=\left|\frac{a_{i n}^{+}}{a_{\text {in }}^{-}} \frac{i \delta}{i \delta-2 \gamma}\right|^{2}
\end{aligned}
$$

A notable result is the reflectivity of the backward direction with $\delta=0$

$$
R_{+}=\left(\frac{g_{r}}{\gamma}\right)^{2}=\left(\frac{g_{r}}{\kappa+2 g_{r}}\right)^{2}
$$

which is not trivial because of the asymmetric backward coupling in the microcavities. In the case of input signal from forward direction, efficiency back coupling from CW to CCW mode constructs a partial stationary mode in the cavity (Fig. 3(a)). Then a relative high reflection of $R_{+} \approx 10 \%$ in on-resonate situation (Fig. 3 (d) blue line), which is coincident with Eq. (19). Therein the loss of the mode (Fig. 2(d)) is $\gamma / 2 \pi=108 \mathrm{GHz}$. When $g_{i}=0$, the separation of two modes in Fig. 2(c) is $2 g_{r} / 2 \pi \approx 68.5 \mathrm{GHz}$. Substituting them into Eq. (19), we get $R_{+} \approx 10 \%$ consistent with our numerical results. As shown in Fig. 3(d), bandwidth of chirality is about $50 \mathrm{GHz}$ in terms of reflection, which is half of the resonance line width. Higher reflection can be achieved by increasing coupling strength $g_{r}$, and the maximum achievable $R_{+}=1 / 4$ for $g_{r} / \kappa=\infty$. In addition, we also check the chiral symmetry breaking for $m=7$ mode. Due to the mismatch of energy, reflection is negligible (Figs. 3(b) and (d) insert). In backward input situation, reflection is negligible as well (Figs. 3(c) and (d) red line).

\section{DISCUSSION}

It is possible to demonstrate the proposed structure in experiment by fabricating nanostrucutres on the top of a on-chip micro-ring resonator. A similar technique had been demonstrated in Ref. [28]. It is also possible to demonstrate the idea in a microsphere or microtoroid cavity, by putting absorptive metal nanoparticle on the surface to induce dissipative coupling, and putting a dielectric nanoparticle or nanotip to the surface to induce reactive coupling.

A limitation of the approach studied here is the induced dissipation to the system. The symmetry breaking is realized with the penalty that a part of energy losses into environment. For the cavities studied here, the best theoretical asymmetry in spectrum is $1 / 4$ according to Eq. (19). In principle, such limitation can be mitigated by replacing the dissipative coupling with a gaining one, or say, by changing the sign of coupling. In this case, the symmetry breaking is obtained without additional loss.

The results presented in Fig. 2 show similar behaviors observed in the study of parity-time (PT) symmetry $[29,30]$ and exceptional point (EP) [21] in optical cavities. Actually, the perfect chirality is obtained at EP, which is common in non-Hermitian systems. Although the evolution of $\Re \omega$ and $\Im \omega$ show similar splitting phenomena with increasing or decreasing $g_{i} / g_{r}$, the system is intrinsically different from the PT-symmetry models $[29,30]$. In the PT-symmetry models, there is reactive coupling but the two modes have different loss/gain factors. In our model, we have both reactive and dissipative coupling, while the two mode have exactly the same frequency. It is also different from recently proposed and demonstrated anti-PT symmetry [31, 32], where they have a pure dissipative coupling but have different mode frequencies.

As described in Sec. II, because the principle for breaking the symmetry is general, it can be applied to any coordinate $\zeta$. One straightforward generalization is breaking the forward and backward symmetry for wave propagating in two coupled straight waveguides. The wave is not limited to be light. It can be any coherent wave source, such as microwave, acoustic wave, spin wave and water wave. Another interesting generalization is breaking time-reversal symmetry, i.e. $\zeta=t$. In this case, if we apply temporal modulations on the dissipative and reactive coupling between systems, the non-reciprocal wave propagation can be realized [24]. We should aware that this approach for non-reciprocity does not conflict with the Lorentz reciprocal theorem, because the temporal modulations are nonlinear effects which lead to the nonreciprocity.

\section{CONCLUSION}

In summary, an approach to break the chiral symmetry of light in a traveling wave microresonator is proposed 
and verified by numerical simulations. Though the discussion is focusing on the chiral symmetry breaking in coordinate $\theta$, the same concept can be used to break symmetry in coordinate $x$ if we straighten the ring into a straight waveguide. This concept also can be used to break time symmetry or others if we introduce two modulations on $t$ or other physical quantities. In terms of $t$, a modulation on the refractive index by $t$ in the entirety system together with another modulation in phase and with the same magnitude modulation on loss (gain) can break the time symmetry.

\section{ACKNOWLEDGMENTS}

We thank Ming Li, Yan-Lei Zhang, Yong Yang, Jianming Wen, and Liang Jiang for fruitful discussion. The work was supported by the Strategic Priority Research Program (B) of the Chinese Academy of Sciences (Grant No. XDB01030200), National Basic Research Program of China (Grant Nos. 2011CB921200 and 2011CBA00200) and the National Natural Science Foundation of China (Grant No. 61505195). F.J.S. is supported by Program for Innovative Research Team (in Science and Technology) in University of Henan Province (IRTSTHN No. 16IRTSTHN028), the State Scholarship Fund from China Scholarship Council (No.201508410405), and the Young Core Instructor Foundation from the Education Department of Henan Province, China (2013GGJS-163).
[1] A. Chiasera, Y. Dumeige, P. Féron, M. Ferrari, Y. Jestin, G. Nunzi Conti, S. Pelli, S. Soria, and G. Righini, Laser Photon. Rev. 4, 457 (2010).

[2] M. R. Foreman, J. D. Swaim, and F. Vollmer, Adv. Opt. Photonics 7, 168 (2015).

[3] S. Yang, Y. Wang, and H. Sun, Adv. Opt. Mater. 3, 1136 (2015).

[4] T. J. Kippenberg, S. M. Spillane, and K. J. Vahala, Opt. Lett. 27, 1669 (2002).

[5] X.-W. Wu, C.-L. Zou, J.-M. Cui, Y. Yang, Z.-F. Han, and G.-C. Guo, J. Phys. B 42, 085401 (2009).

[6] B. Redding, L. Ge, Q. Song, J. Wiersig, G. S. Solomon, and H. Cao, Phys. Rev. Lett. 108, 253902 (2012).

[7] G. Chern, H. Tureci, A. D. Stone, R. Chang, M. Kneissl, and N. Johnson, Appl. Phys. Lett. 83, 1710 (2003).

[8] Q. Song, N. Zhang, H. Zhai, S. Liu, Z. Gu, K. Wang, S. Sun, Z. Chen, M. Li, and S. Xiao, Sci. Rep. 4, 06493 (2014).

[9] J. Wiersig and M. Hentschel, Phys. Rev. Lett. 100, 033901 (2008).

[10] J. Zhu, Ş. Özdemir, L. He, and L. Yang, Opt. Express 18, 23535 (2010).

[11] J. Wiersig, Phys. Rev. A 84, 063828 (2011).

[12] M. Kim, K. Kwon, J. Shim, Y. Jung, and K. Yu, Opt. Lett. 39, 2423 (2014).

[13] J. Wiersig, Phys. Rev. Lett. 112, 203901 (2014).

[14] J. Wiersig, Phys. Rev. A 93, 033809 (2016).

[15] M. Terrel, M. J. Digonnet, and S. Fan, Laser Photonics Rev. 3, 452 (2009).

[16] L. Ge, R. Sarma, and H. Cao, Phys. Rev. A 90, 013809 (2014).

[17] D. L. Sounas, C. Caloz, and A. Alù, Nat. Commun. 4 (2013).
[18] C.-H. Dong, Z. Shen, C.-L. Zou, Y.-L. Zhang, W. Fu, and G.-C. Guo, Nat. Commun. 6 (2015).

[19] J. Kim, M. C. Kuzyk, K. Han, H. Wang, and G. Bahl, Nat. Phys. 11, 275 (2015).

[20] R. Sarma, L. Ge, J. Wiersig, and H. Cao, Phys. Rev. Lett. 114, 053903 (2015).

[21] B. Peng, Ş. Özdemir, S. Rotter, H. Yilmaz, M. Liertzer, F. Monifi, C. Bender, F. Nori, and L. Yang, Science 346, 328 (2014).

[22] M. Golshani, S. Weimann, K. Jafari, M. K. Nezhad, A. Langari, A. Bahrampour, T. Eichelkraut, S. Mahdavi, and A. Szameit, Phys. Rev. Lett. 113, 123903 (2014).

[23] S. Longhi, Opt. Lett. 40, 1278 (2015).

[24] A. Metelmann and A. Clerk, Phys. Rev. X 5, 021025 (2015).

[25] M. D. Baaske, M. R. Foreman, and F. Vollmer, Nature nanotechnology 9, 933 (2014).

[26] B.-Q. Shen, X.-C. Yu, Y. Zhi, L. Wang, D. Kim, Q. Gong, and Y.-F. Xiao, Phys. Rev. Appl. 5, 024011 (2016).

[27] B. E. Saleh, M. C. Teich, and B. E. Saleh, Fundamentals of photonics, Vol. 22 (Wiley New York, 1991).

[28] L. Feng, Z. J. Wong, R.-M. Ma, Y. Wang, and X. Zhang, Science 346, 972 (2014).

[29] L. Chang, X. Jiang, S. Hua, C. Yang, J. Wen, L. Jiang, G. Li, G. Wang, and M. Xiao, Nat. Photonics 8, 524 (2014).

[30] B. Peng, a. K. Özdemir, F. Lei, F. Monifi, M. Gianfreda, G. L. Long, S. Fan, F. Nori, C. M. Bender, and L. Yang, Nat. Phys. 10, 394 (2014).

[31] L. Ge and H. E. Türeci, Phys. Rev. A 88, 053810 (2013).

[32] P. Peng, W. Cao, C. Shen, W. Qu, J. Wen, L. Jiang, Y. Xiao, and N. Haven, arXiv , 1509.07736. 